\title{
Surgical Treatment of Hip Instability in Patients With Lower Lumbar Level Myelomeningocele: Is Muscle Transfer Required?
}

\author{
Timur Yildirim MD, Sarper Gursu MD, \\ Illhan Avni Bayhan MD, Hakan Sofu MD, \\ Aysegul Bursali MD
}

Published online: 25 April 2015

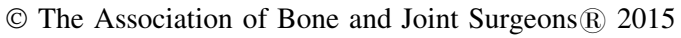

\begin{abstract}
Background Treatment of hip instability in patients with lower lumbar level myelomeningocele is clinically challenging. Muscle transfer procedures, release of contractures, and intertrochanteric varus-rotation osteotomies have been described to restore weak or absent abductor strength as well as relocation of the hip. However, controlled trials evaluating hip instability in lower lumbar myelomeningocele are limited in the current literature.

Questions/purposes The purposes of this study were to compare the (1) radiographic evidence for joint stability; (2) clinical outcomes (including abductor strength,
\end{abstract}

Each author certifies that he or she, or a member of his or her immediate family, has no funding or commercial associations (eg, consultancies, stock ownership, equity interest, patent/licensing arrangements, etc) that might pose a conflict of interest in connection with the submitted article.

All ICMJE Conflict of Interest Forms for authors and Clinical Orthopaedics and Related Research ${ }^{\circledR}$ editors and board members are on file with the publication and can be viewed on request.

Each author certifies that his or her institution approved the human protocol for this investigation, that all investigations were conducted in conformity with ethical principles of research, and that informed consent for participation in the study was obtained.

This work was performed at the Baltalimani Bone Diseases Training and Research Hospital, Istanbul, Turkey.

T. Yildirim $(\bowtie)$, S. Gursu, İ. A. Bayhan

Baltalimani Bone Diseases Training and Research Hospital,

Rumeli Hisari Cad. No: 62, Sariyer/Istanbul, Turkey

e-mail: drtimur@hotmail.com

H. Sofu

Orthopaedic and Traumatology Department, Faculty of

Medicine, Erzincan University, Erzincan, Turkey

A. Bursali

Istanbul, Turkey ambulatory ability, and residual use of orthoses); and (3) complications between patients undergoing combined periarticular contracture releases and bony procedures with and without external oblique abdominal muscle transfers. Methods Between 2004 and 2013, 14 pediatric patients (16 hips) were treated for hip instability secondary to myelomeningocele using releases with or without muscle transfer. From those, 13 patients ( 15 hips) with a mean age of 6 years who had L3 to L5 level involvement were evaluated retrospectively. The patients were separated into two groups. Nine hips (in eight patients) were treated by performing a combination of external oblique abdominal muscle transfer to the greater trochanter, periarticular release of contractures, and bony procedures. These were compared with six hips (five patients) treated by performing a combination of periarticular release of contractures and bony procedures without external oblique abdominal muscle transfer. This study compared the results between two surgeons, one of whom always performed these muscle transfers in this setting and the other who never performed muscle transfer during the study period. The patients were clinically followed up at a mean of 41 months (range, 14-122 months); none of the patients was lost to followup. Radiographic evaluation criteria included Reimer's migration index, acetabular index, femoral neck-shaft angle, pelvic obliquity, and the presence of scoliosis. Clinical evaluation included muscle strength examination, periarticular contractures, necessity for using orthoses as walking aids, and Hoffer criteria of mobility. All complications were also noted from a chart review.

Results There were no differences between the two groups regarding postoperative femoral head localization, reflecting the presence or absence of residual subluxation, according to Reimer's index (Reimer's index $=32 \%$; range, $10 \%-40 \%$, in the muscle transfer group compared 
with $27 \%$; range, $15 \%-43 \%$, in the no-transfer group at latest followup; $\mathrm{p}=0.723$ ). Postoperatively, abductor strength improved in the group treated with external oblique transfer compared with the group treated without muscle transfer (median improvement of 2 versus 0 , $\mathrm{p}=0.02$ ), but this improvement neither resulted in a clinically important difference in Hoffer criteria of mobility (no change was detected in either group) nor decreased the need for use of an orthosis (no change was detected in either group). With the numbers available, there was no difference with respect to complications between the two groups (two complications versus one).

Conclusions External oblique abdominal muscle transfer did not provide a clinically important improvement in functional recovery when compared with patients with L3 to L5 myelomeningocele and hip instability who were treated without it. We therefore do not recommend a routine muscle transfer procedure during the operative management of hip instability in patients with lower lumbar level myelomeningocele.

Level of Evidence Level III, therapeutic study.

\section{Introduction}

Myelomeningocele, which mainly affects the musculoskeletal system as well as causing disturbances in cognitive functions and genitourinary and gastrointestinal systems, is a complex congenital disorder that can lead to life-long disability. Hip problems restricting the ambulatory capacity of the patient mostly occur as a result of instability together with varying degrees of muscle contractures causing pelvic obliquity and scoliosis [17]. Pelvic ring muscles do not function properly in patients with upper-level lesions and thus reduction of the hip generally ends up with high failure rates in such patients. By contrast, treatment of hip instability in patients with sacral-level lesion is generally similar to developmental dysplasia. Hip deformities and contractures secondary to L3 or lower lumbar level myelomeningocele in patients with high expected long-term functional mobilization capacity but weak abductor and extensor muscle strength of the hip as well as forceful abductor and flexor muscles leading to hip instability at the same time may be treated more aggressively.

Operative management of hip disorders in lower lumbar myelomeningocele as well as the most reliable surgical technique is still controversial. Surgical techniques including iliopsoas and external oblique abdominal muscle transfers to obtain a stable joint during the treatment of hip dislocations have been described $[1,3,14,16]$. As an alternative to muscle transfer, surgical release of the muscle contractures in combination with bony procedures in the treatment of hip instability was also reported as effective to obtain enough stability by a limited number of publications in the literature [6, 18]. However, because of methodological differences like nonstandardized surgical techniques or differences in the neurological level, the available studies about these treatment options can be difficult to compare against one another.

The main purpose of our study was to compare the (1) radiographic evidence for joint stability; (2) clinical outcomes (including abductor strength, ambulatory ability, and residual use of orthoses); and (3) complications between patients undergoing combined periarticular contracture releases and bony procedures with and without external oblique abdominal muscle transfers.

\section{Patients and Methods}

Clinical data of the patients who were surgically treated between 2004 and 2013 with the diagnosis of hip subluxation or dislocation secondary to lower lumbar myelomeningocele were retrospectively evaluated after having approval from the institutional review board. The patients with L3 to L5 level lesions, who were able to walk independently, have quadriceps muscle strength of at least $3 / 5$, and who did not have previous hip surgery were included in our study. Teratologic hip dislocations were excluded from the study. The indication for hip relocation surgery was including ambulatory patients who had hip subluxation or dislocation with L3 to L5 level of involvement and at least $3 / 5$ quadriceps muscle strength. Demographic data, ambulatory functions, and radiographic changes were comparatively analyzed. From 14 patients (16 hips), 13 patients (15 hips) (five boys, eight girls) who met the study inclusion criteria were clinically evaluated (Table 1). One patient was excluded from the study as a result of L2 level involvement. The mean age at the time of surgery was 6 years (range, 21 months to 12 years). The patients were separated into two groups according to surgical technique preferred by two different orthopaedic surgeons (AB performed the muscle transfer; TY performed the hip surgeries without muscle transfer) (Table 2). This study compared the results between two surgeons, one of whom always performed these muscle transfers in this setting and the other who never performed musle transfer during the study period. Nine hips (in eight patients) were treated by performing a combination of external oblique abdominal muscle transfer to trochanter major with or without femoral osteotomy, acetabuloplasty, and release of periarticular muscle contractures (Fig. 1AB). Another six hips (in hip patients) were treated by performing a combination of femoral osteotomy and/or acetabuloplasty and release of periarticular muscle contractures without any muscle transfer procedure (Fig. 2A-B). 


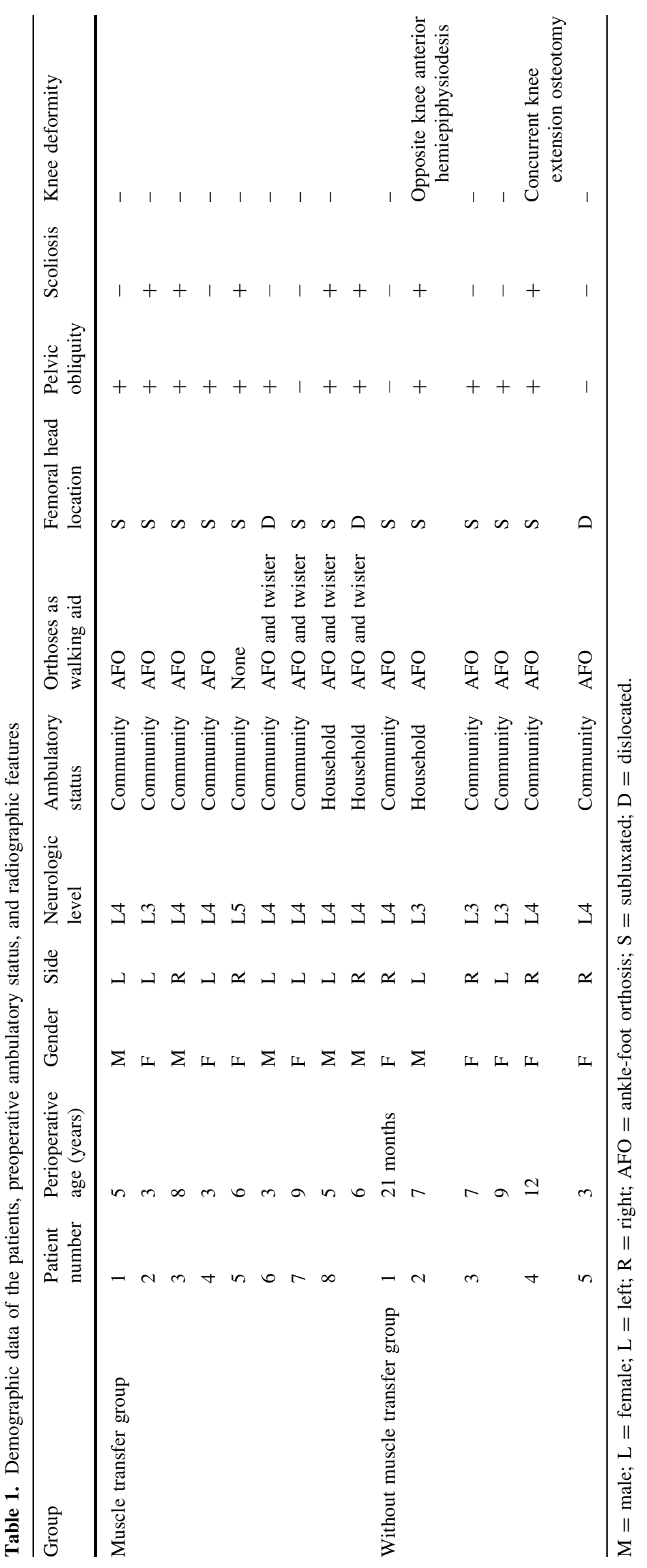


Table 2. Type of surgical procedures $(\mathrm{N}=15)$

\begin{tabular}{|c|c|c|c|}
\hline Treatment groups & Surgical procedures & Number of hips & Total \\
\hline \multirow[t]{2}{*}{ Muscle transfer group } & $\begin{array}{l}\text { Acetabuloplasty }+ \text { soft tissue release }+ \text { external oblique abdominal } \\
\text { muscle transfer to great trochanter }\end{array}$ & 6 & 9 \\
\hline & $\begin{array}{l}\text { Acetabuloplasty }+ \text { soft tissue release }+ \text { external oblique abdominal } \\
\text { muscle transfer to great trochanter }+ \text { femoral osteotomy }\end{array}$ & 3 & \\
\hline \multirow[t]{2}{*}{ Without transfer group } & Acetabuloplasty + soft tissue release & 3 & 6 \\
\hline & Acetabuloplasty + soft tissue release + femoral osteotomy & 3 & \\
\hline
\end{tabular}

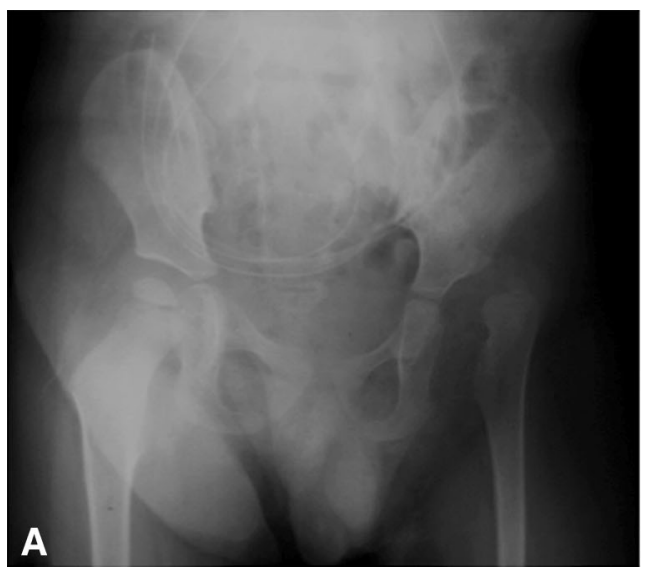

Fig. 1A-B A 3-year-old male patient with L4-level myelomeningocele was treated by performing a combination of acetabuloplasty, release of iliopsoas and adductor tendons, and external oblique

One patient in each group had bilateral surgical intervention. Twelve hips were subluxated, and three hips were dislocated preoperatively. The median followup was 36 months (range, 14-122 months) in the muscle transfer group and 40 months (range, 19-66 months) in the group without muscle transfer. None of the patients were lost during followup.

With the numbers available, there were no differences between the study groups in terms of mean age at the time of surgery, level of neurological involvement, localization of the femoral head, abductor strength of the hip, and mobilization capacity of the patients between the two groups preoperatively $(\mathrm{p}>0.05)$.

The acetabuloplasty technique was identical in all hips and it was performed as lateral acetabular osteotomy described by Tönnis et al. [15]. The decision about femoral osteotomy was made according to the presence of coxa valga and/or abnormally excessive femoral anteversion preoperatively. An iliopsoas tenotomy was performed at the level of the lesser trochanter if a hip flexion contracture of more than $20^{\circ}$ was identified preoperatively [5]. Level of

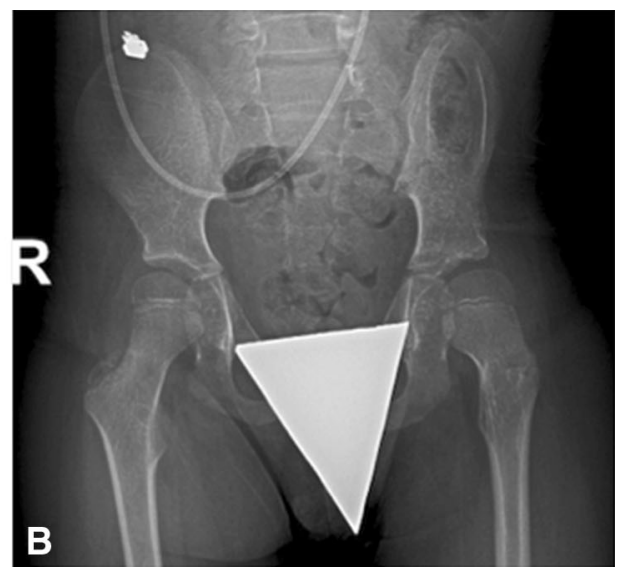

abdominal muscle transfer to trochanter major. Dislocated left hip in preoperative radiograph (A) and after 5 years of followup (B).
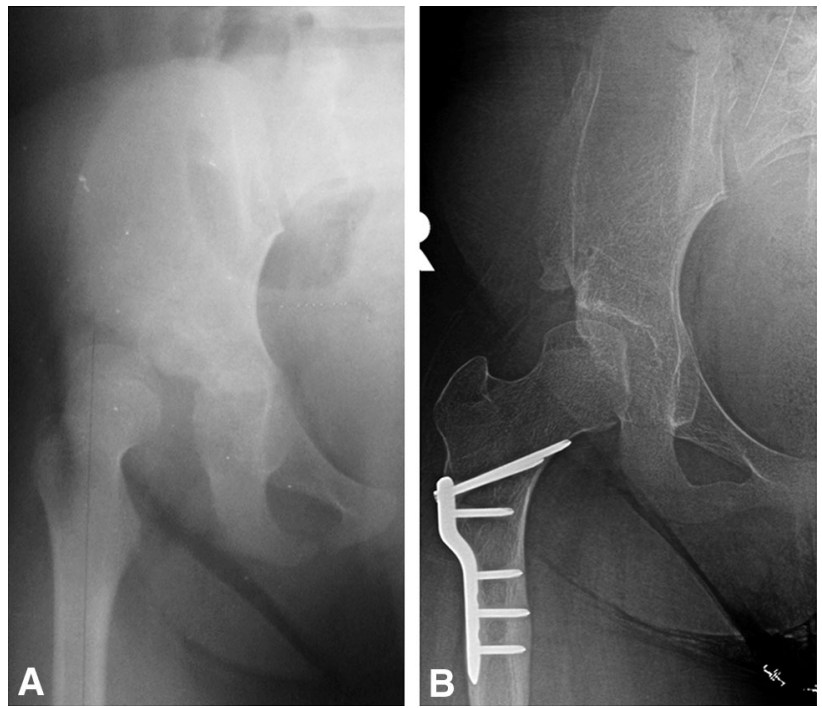

Fig. 2A-B A 7-year-old female patient with L3-level myelomeningocele was treated by performing a combination of acetabuloplasty, proximal femoral varus-derotation osteotomy, and release of periarticular contractures. Subluxated right hip on preoperative radiograph (A) and after 46 months of followup (B). 
Table 3. Radiographic findings

\begin{tabular}{|c|c|c|c|c|c|c|c|}
\hline \multirow[t]{2}{*}{ Measured parameters } & \multicolumn{3}{|c|}{ Muscle transfer group } & \multicolumn{3}{|c|}{ Without muscle transfer group } & \multirow[t]{2}{*}{$\mathrm{p}$ value } \\
\hline & Median & Minimum & Maximum & Median & Minimum & Maximum & \\
\hline Reimer's index-preoperative (\%) & 80 & 45 & 100 & 53 & 34 & 100 & 0.097 \\
\hline Reimer's index-latest followup (\%) & 32 & 10 & 40 & 27 & 15 & 43 & 0.723 \\
\hline Acetabular index angle-preoperative (degrees) & 35 & 16 & 51 & 32 & 26 & 41 & 0.637 \\
\hline Acetabular index angle—-latest followup (degrees) & 21 & 11 & 40 & 19 & 15 & 25 & 0.858 \\
\hline Femoral neck shaft angle—preoperative (degrees) & 150 & 134 & 162 & 148 & 138 & 150 & 0.374 \\
\hline Femoral neck shaft angle—latest followup (degrees) & 140 & 124 & 152 & 132 & 121 & 151 & 0.216 \\
\hline
\end{tabular}

neurologic involvement was determined according to Sharrard's system [13]. According to this system, three patients had L3, nine patients had L4, and one patient had a L5 level lesion. From the chart review functional ambulatory status of the patients was classified preoperatively into four groups as mobile in community, mobile inside home, nonfunctional ambulatory, and nonambulatory according to the criteria described by Hoffer et al. [10]. One patient with L3 level neurologic involvement and one patient with L4 level neurologic involvement were mobile inside the home, whereas all other patients were mobile in the community.

A hip spica cast was applied to all patients after surgery and removed at the end of 6 weeks postoperatively. Physical therapy and a rehabilitation program were routinely started at the end of 6 weeks immediately after cast removal.

ROM in the hips and fixed contractures were assessed by using a goniometer during physical examination by the primary investigator (TY). More than $20^{\circ}$ of flexion contracture of the hip and more than $10^{\circ}$ of limitation in ROM either in the coronal or horizontal plane were recorded as signs of fixed contracture. Muscle strengths were evaluated manually during physical examination. All complications were noted from a chart review.

Radiographic assessment of the hip was performed preand postoperatively by the primary investigator (TY) for the measurement of femoral head coverage (Reimer's index), acetabular index angle, Shenton line orientation, and femoral neck-shaft angle on the plain pelvic roentgenograms obtained when the patient was lying supine. Coronal and sagittal plane deformities of the vertebral column as well as pelvic obliquity were assessed on two-plane standing spinal orthoroentgenograms. Reimer's index measurement between $33 \%$ and $99 \%$ was accepted as subluxation and $\geq 100 \%$ was accepted as dislocation of the hip. Hips with a Reimer's index $>33 \%$ during postoperative followup were defined as recurrence of instability.

Clinical and radiological data of our study were analyzed by using the SPSS 20.0 computer program (SPSS Inc, IBM, Chicago, IL, USA). Categorical data of treatment groups were assessed by chi square test. Wilcoxon signedrank test was applied to compare related data of preoperative and postoperative periods within each group. The nonparametric Mann-Whitney U-test was applied to make comparison of independent variables between the two groups. The level of significance was set at $\mathrm{p}<0.05$.

\section{Results}

There was no difference between the two groups regarding postoperative femoral head localization according to Reimer's index (Reimer's index $=32 \%$; range, $10 \%-40 \%$, in the muscle transfer group compared with $27 \%$; range, $15 \%-43 \%$, in the no-transfer group at latest followup; $\mathrm{p}=0.723$ ) (Table 3). Residual subluxation was detected in five hips postoperatively, three of which were from patients treated with external oblique muscle transfer and the other two from without muscle transfer. The mean Reimer's index measurement of these five hips was $61 \%$ preoperatively and $39 \%$ postoperatively. We did not detect progressive loss of the improvement obtained by surgical intervention in any of the hips. No dislocation was observed in any of the hips during the postoperative followup period. Pelvic obliquity also improved when both groups were evaluated together, but there was no difference between the groups in comparison according to improvement in pelvic obliquity.

Functional ambulatory status determined according to Hoffer et al. did not show an increase in our patients regardless of the preferred surgical technique; seven patients were community ambulators and one was a household ambulatory in the muscle transfer group, whereas in the no muscle transfer group, four patients were community ambulators and one was a household ambulator $(\mathrm{p}=1)$. Although abductor strength was greater in the group treated with transfer and did not improve in the group treated without transfer (median improvement was 2 versus 0 , $\mathrm{p}=0.02$ ), this difference did not result in a clinically important benefit, because limping resulting from abductor insufficiency remained in both groups after surgery, and 
there was no decrease in the need for walking orthoses after surgical treatment in either group. Eight hips in patients treated with external oblique muscle transfer, which consisted of hips treated by performing combined surgical intervention with external oblique abdominal muscle transfer, demonstrated an increase in abductor muscle strength postoperatively.

We did not observe any wound infections, nonunions of the osteotomies, or iatrogenic joint stiffness during the postoperative followup period. In patients treated with external oblique muscle transfer, one patient sustained a fracture of the distal femoral metaphysis, and another had a distal radial fracture, whereas one patient without muscle transfer had a tibial shaft fracture within the first 6 months after surgery. These fractures were successfully treated without surgery. The overall frequency of complications was not different with the numbers available between the groups $(\mathrm{p}=1)$.

\section{Discussion}

Hip problems are frequently seen in patients with myelomeningocele and the treatment of these problems reveals various difficulties for orthopaedic surgeons. Surgical treatment of hip instabilities occurring as a result of muscle imbalance around the joint in myelomeningocele and the most effective surgical method to obtain a permanent stability of the joint are controversial. Although various surgical techniques that mainly aim for correction of muscular imbalance such as tenotomy of the iliopsoas and adductor muscles, transfer of the iliopsoas, or external oblique abdominal muscles as well as different types of osteotomies to correct secondary adaptive bony deformities have been described [2, 9, 12, 19]; the number of clinical trials comparing the results of hip reconstruction methods in lower lumbar myelomeningocele are limited in the literature. Our study demonstrated that there are no clinically important differences in the results of surgical hip reconstruction performed with or without external oblique abdominal muscle transfer in the aspects of clinical and radiological outcomes as well as the complication rates. Although increased abductor strength of the hip was obtained in the muscle-transfer group, this increase did not appear to improve the clinical status and daily living of the patients.

There are some limitations of our study. First, because of the retrospective nature of the study and also because of the limited number of patients, analysis of the variables such as femoral osteotomy and iliopsoas tenotomy could not be done; that may have affected the results between the subgroups. Second, muscle strength was examined manually. Evaluation of the muscle transfer with electromyography or a quantitative muscle strength test would have given more objective data. Third, one patient in the muscle-transfer group had involvement of the L5 nerve root. L5 involvement differs from L3 and L4 levels because of differences in muscle strength, and unlike L3 and L4 level, most of the L5-level patients are able to ambulate without any walking aids over the longer term. As such, longer-term followup of the cohort will be important. Last, because of the small sample size, common complications may have not been detected. Although our patient numbers were adequate to demonstrate differences and similarities between the two treatment groups, a larger sample size would permit a more accurate quantification of potentially important differences.

We found that the radiographic appearance of the hips treated with external oblique transfer was better than those treated without a transfer but that this difference did not result in functionally important improvements in those patients. Dias and Hill [6] mentioned that sustained joint stability could be achieved in 23 of 27 hips after intertrochanteric varus and derotation osteotomy; hence, iliopsoas muscle transfer was not necessary. They also concluded that the main reason of recurrent instability in three of four hips with unsatisfactory results in their study was the acetabular index exceeding $25^{\circ}$ postoperatively. In their study comparing iliopsoas muscle transfer and combined varus-rotation osteotomy, Weisl et al. [18] reported that a contained hip was obtained in $54 \%$ of the muscle transfer group and $70 \%$ of the osteotomy group.

The main purpose of muscle transfer procedures performed during the surgical treatment of hip instability in patients with lower lumbar myelomeningocele is to achieve improvement of ambulatory functions by restoring the abductor lever arm and contributing to the establishment of sustained joint stability. In the present study, although abductor muscle strength improved in the external oblique abdominal muscle transfer group, we could not detect any improvement in the Trendelenburg sign and limping pattern in the patients treated with muscle transfer compared with those treated without muscle transfer. Improvement rates of resistance to gravity were reported as $9 \%$ and $28 \%$ in two different studies evaluating iliopsoas tendon transfer to the posterior part of the trochanter major [3, 11]. Tosi et al. [16] concluded that although hip stability could be achieved by performing external oblique abdominal muscle transfer, its contribution to abductor muscle strength was very limited. Muscle strength improvements, which are insufficient to provide active motion against gravity, cannot create a positive effect on functional status. Because previous studies in the literature lack a standardized method in muscle strength evaluation, it is impossible to make an accurate comparison among the outcomes of different treatment methods. However, long-term results demonstrate that independently from 
the surgical technique, muscle transfers do not provide any decrease or change in the need of walking orthoses as well as the walking pattern. In a comparison of the functional results of the patients who had surgical reduction with those who did not, Feiwell et al. [7] showed that surgical reduction neither reduced the need for brace nor improved hip ROM and mobility. Prior work suggests that muscle transfer surgery increases the energy consumption, and in unilateral hip instability, for symmetry, contracture release surgery has been proposed rather than joint reduction procedures [8]. Correll and Gabler [4] described that hip contracture release is a safe procedure with a low complication rate, which also permits early postoperative mobilization.

With the numbers available, we found no difference in the frequency or severity of complications between patients treated with and without muscle transfer. Joint stiffness, pathological fractures, avascular necrosis, redislocation, and results of this progressive motor functional loss were described after muscle transfer surgeries. In the study of Carroll and Sharrards, the long-term followup of 53 patients with posterior iliopsoas muscle transfer showed $3.4 \%$ of infection and $25 \%$ of avascular necrosis. They observed pathological femur fractures related to osteopenia caused by long-term immobilization [3]. In the study of Weisl et al., comparison of posterior iliopsoas transfer with varus derotational osteotomy showed high incidence of pathological femur fractures and joint stiffness in both groups [18]. Dias and Hill performed varus derotation intertrochanteric osteotomy for subluxation in 27 hips. They observed $20 \%$ of femur fractures and neither avascular necrosis nor hip stiffness was detected. They mentioned that intertrochanteric varus osteotomy is easy to perform without causing hip stiffness and decreasing hip flexion power [6]. Although we found no differences in complications, our study was small, and we believe that if the larger procedure (muscle transfer) does not add a functionally evident benefit, patients are better treated without the additional surgery involved with muscle transfer.

In conclusion, surgical treatment including femoral and/ or acetabular osteotomies combined with release of the muscle contractures revealed similar clinical and radiological outcomes when compared with the same surgical interventions combined with an external oblique abdominal muscle transfer. Therefore, we do not recommend routine muscle transfer procedures during the operative management of hip instability in patients with lower lumbar level myelomeningocele. The present study may be a reference for long-term clinical trials with larger cohorts to evaluate the treatment of patients with similar problems.
Acknowledgments We thank Selami Sozubir for his guidance in development of the surgical technique.

\section{References}

1. Bazih J, Gross RH. Hip surgery in the lumbar level myelomeningocele patient. J Pediatr Orthop. 1981;1:405-411.

2. Breed AL, Healy PM. The midlumbar myelomeningocele hip: mechanism of dislocation and treatment. J Pediatr Orthop. 1982;2: $15-24$.

3. Carroll NC, Sharrard WJ. Long-term follow-up of posterior iliopsoas transplantation for paralytic dislocation of the hip. J Bone Joint Surg Am. 1972;54:551-560.

4. Correll J, Gabler C. The effect of soft tissue release of the hips on walking in myelomeningocele. J Pediatr Orthop B. 2000;9:148153.

5. Dias LS. Evaluating gait in myelomeningocele. In: Sarwark JF LJ, ed. Caring for the Child With Spina Bifida. Rosemont, IL, USA: American Academy of Orthopaedic Surgeons; 2001:263-268.

6. Dias LS, Hill JA. Evaluation of treatment of hip subluxation in myelomeningocele by intertrochanteric varus derotation femoral osteotomy. Orthop Clin North Am. 1980;11:31-37.

7. Feiwell E, Sakai D, Blatt T. The effect of hip reduction on function in patients with myelomeningocele. Potential gains and hazards of surgical treatment. J Bone Joint Surg Am. 1978;60:169-173.

8. Gabrieli AP, Vankoski SJ, Dias LS, Milani C, Lourenco A, Filho JL, Novak R. Gait analysis in low lumbar myelomeningocele patients with unilateral hip dislocation or subluxation. $J$ Pediatr Orthop. 2003;23:330-334.

9. Gugenheim JJ, Rosenthal RK, Dabrowski S, Hall JE. The adductor transfer in the high-risk hip in myelodysplasia: a preliminary report. Clin Orthop Relat Res. 1978;132:108-114.

10. Hoffer MM, Feiwell E, Perry R, Perry J, Bonnett C. Functional ambulation in patients with myelomeningocele. J Bone Joint Surg Am. 1973;55:137-148.

11. Lee EH, Carroll NC. Hip stability and ambulatory status in myelomeningocele. J Pediatr Orthop. 1985;5:522-527.

12. Phillips DP, Lindseth RE. Ambulation after transfer of adductors, external oblique, and tensor fascia lata in myelomeningocele. J Pediatr Orthop. 1992;12:712-717.

13. Sharrard WJ. The segmental innervation of the lower limb muscles in man. Ann R Coll Surg Engl. 1964;35:106-122.

14. Stillwell A, Menelaus MB. Walking ability after transplantation of the iliopsoas. A long-term follow-up. J Bone Joint Surg Br. 1984;66:656-659.

15. Tönnis D, Briining K, Heinecke A. Lateral acetabular osteotomy. J Pediatr Orthop B. 1994;3:40-46.

16. Tosi LL, Buck BD, Nason SS, McKay DW. Dislocation of hip in myelomeningocele. The McKay hip stabilization. J Bone Joint Surg Am. 1996;78:664-673.

17. Trivedi J, Thomson JD, Slakey JB, Banta JV, Jones PW. Clinical and radiographic predictors of scoliosis in patients with myelomeningocele. J Bone Joint Surg Am. 2002;84:1389-1394.

18. Weisl H, Fairclough JA, Jones DG. Stabilisation of the hip in myelomeningocele. Comparison of posterior iliopsoas transfer and varus-rotation osteotomy. J Bone Joint Surg Br. 1988;70: 29-33.

19. Yngve DA, Lindseth RE. Effectiveness of muscle transfers in myelomeningocele hips measured by radiographic indices. J Pediatr Orthop. 1982;2:121-125. 